\title{
Smart Hybrid Power System for Base Transceiver Stations with Real-Time Energy Management
}

\author{
Panagiotis D. Diamantoulakis \\ Department of Electrical Engineering \\ Aristotle University of Thessaloniki \\ Thessaloniki, Greece \\ padiaman@auth.gr
}

\author{
A. Ghassemi \\ Department of Electrical Engineering \\ University of Victoria \\ Victoria, BC Canada \\ aghassem@ece.uvic.ca
}

\author{
George K. Karagiannidis \\ Department of Electrical Engineering \\ Aristotle University of Thessaloniki \\ Thessaloniki, Greece \\ geokarag@auth.gr
}

\begin{abstract}
Reducing the power consumption of base transceiver stations (BTSs) in mobile communications networks is typically achieved through energy saving techniques, where they can also be combined with local power generators to create a hybrid power system (HPS). Such a system has reduced power consumption and operational cost, without taking the advantage of real-time energy management. In this paper, we introduce the smart HPS that can facilitate energy consumption scheduling (ECS) via an intelligent connection to the power grid. In doing so, we first develop sensor control and communication systems with an embedded smart ECS unit for the HPS. Then, we propose a real-time energy management algorithm to reduce the operational cost of BTS, according to real-time pricing and estimating demand and supply. The numerical results presented show a significant reduction in the BTS operational costs. We also develop a techno-economic and sizing analysis to describe the total cost of the smart HPS taking the real-time energy pricing into account. Since the lifetime of the operating system can be quite long, our results show that there is possibility to make a profit.
\end{abstract}

\section{INTRODUCTION}

Recent years have seen a surge in research on the power consumption aspect of wireless and cellular networks due to the increasing concern on rising global energy demand and decreasing the industry's overall carbon footprint. The power consumption of cellular networks also constitutes a large portion of operating expenses for service providers [1], [2]. Within a cellular network, the power needed to run a base transceiver station (BTS) as well as the corresponding cooling facilities forms the major share of energy consumption. This increases the motivation for the development of BTS energy savings techniques and/or finding new solutions for the uninterruptable and alternative BTS power supplies.

Previous methods for reducing the power consumption of a BTS can be largely classified into the next four categories [2]. One approach is to relocate the radio frequency (RF) converters and power amplifiers from the base of the station to the top of the tower close to the antenna and connecting them via fiber cables [3]. The second method is based on exploiting the economy mode (ECO) feature, e.g. turning the transceivers on/off during low traffic conditions [3], to improve BTS energy efficiency. The third possibility is to use advanced climate control for air conditioners to conserve power and/or using passively-cooled cabinets [4]. The last scheme is based on using higher efficiency rectifiers as part of BTS installations [2].

Renewable energy sources such as wind and solar power represent attractive and economic solutions for alternative BTS energy sources. These resources jointly with non renewablebased electricity generators, such as diesel generators and electrical grid, can create a hybrid power system (HPS) to provide a more reliable power supply for BTSs, while reducing the operational cost of BTSs. As examples of such systems, [5], [6] and subsequently [7] have proposed a hybrid solarwind-batteries-diesel/electric grid system that can substantially reduce the operational cost. In [7] a time-step algorithm has also been proposed to size properly the hybrid system, in order to partially- or fully-meet the load demands of a BTS, while evaluating the amount of $\mathrm{CO} 2$ emissions. Moreover, the HPSs require control and communications systems to locally and resourcefully manage supply and demand within a BTS. In this paper, we first develop a sensor control system and communications network to facilitate power measurements from BTS loads and local generators, and provide communications among sensor nodes.

Previously proposed HPSs can work in two disconnected and connected modes of operations, according to measuring shortage and surplus in electrical power. In the former mode, it locally balances supply and demand and if there is a surplus in energy, there is no possibility to transfer/sell this energy to the grid. In the latter mode, the HPS employs the power grid besides local energy resources to balance supply and demand. In this case, there is no capability for energy consumption scheduling (ECS) to facilitate real-time energy management, and consequently reduce further cost of operation. To address these problems, this paper introduces the new concept of the smart hybrid power system (SHPS), based on real-time energy management, in which our main objective is to optimize the cost of BTS operation according to real-time pricing, demand and supply estimation. We thus consider estimating the amount of electricity produced by renewable energy generators over different time slots, in order to schedule energy consumption within a BTS.

With the aim to implement real-time energy management, a SHPS is considered as a combination of local power generators and a smart ECS unit, which can facilitate the selling and 
buying of electricity from the power grid. In this case, the communication network related to the SHPS need to be integrated into a smart grid communications infrastructure in order to provide connectivity with the utility operation center. Since the SHPS has the ability to shift the cooling load into a time slot with a lower price, from utility perspective this is beneficial for mitigating peak demand, while from the mobile operator it is advantageous for reducing the operational cost.

Moreover, we investigate the performance of the proposed method while taking actual real-time price tarrifs. The numerical results show that the proposed technique can significantly reduce operational cost. Finally, we provide a techno-economic analysis, which is based on the algorithm proposed in [7]. This analysis adjusts the total cost of the SHPS according to new factors, such as the cost associated with the sensor network and communications infrastructure, and real-time energy pricing.

The rest of the paper is organized as follows. In Section II the HPSs model and its components are described, including the development of the sensor control system and local wireless sensor network. In Section III the smart hybrid power system and the formulation of the cost optimization problem based on real-time energy management are introduced. In Section IV the techno-economic analysis for the proposed SHPS is presented. Numerical results are discussed in Section $\mathrm{V}$, while Section VI concludes the paper.

\section{A POWER HYBRID SYSTEM FOR BTS}

This section provides an overview of the BTS HPS described in [7], with a focus on its major components and the related energy-efficient BTS operation. We then describe the control system and local communications network required for the hybrid system.

\section{A. Power System Model}

Consider a BTS with a HPS, as illustrated in Fig. 1. This system includes renewable generators, local power generators, energy storage devices, and power consumption model. Renewable energy sources, such as wind and solar, are considered, while energy storage devices and local generators includes batteries and diesel generators.

The power consumption model consists of two major parts. The first part is related to the AC power consumption, a power figure which is mainly consumed in a BTS cooling system. The second part is associated with the DC loads corresponding to radio equipment, such as power amplifiers and mixers/converters. In order to describe the BTS energy saving strategies facilitated by the hybrid power system, we first define variables related to the loads and generators.

Let $P_{B T S}(t)$ represent the total power consumed in the time slot $t \in \mathrm{T}=\{1, \ldots . ., T\}$ via $\mathrm{AC}$ and $\mathrm{DC}$ loads, denoting by $A(t)$ and $D(t)$, respectively. The produced energy by the photo-voltaic (PV) generators and wind turbines are represented as $P_{p} \in \mathrm{P}_{p}=\left\{P_{p}(1), \ldots, P_{p}(T)\right\}$ and $P_{w} \in$ $\mathrm{P}_{w}=\left\{P_{w}(1), \ldots, P_{w}(T)\right\}$, correspondingly. Let $n_{i}, n_{r}$, and $n_{c}$ denote the efficiency of the inverter, rectifier and converter, respectively. $P_{b}(t)$ represents the amount of power consumed

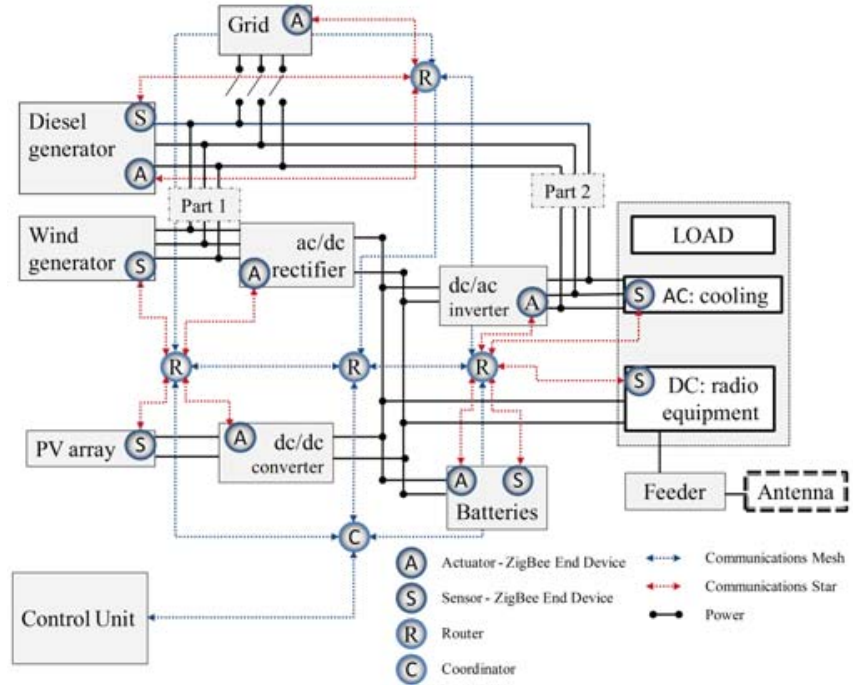

Fig. 1. The HPS including sensor control and communications systems.

or provided by the batteries. If $P_{b}(t)<0$, then the batteries are recharging, while if $P_{b}(t)>0$, then they are supplying energy. The parameter $P_{d}(t)$ also denotes the energy provided by the diesel generator. The amount of energy exchange between the BTS and the grid is defined as $P_{g}(t)$. We assume $P_{g}(t)>0$ or $P_{g}(t)<0$ when the hybrid power system has surplus or shortage in energy. The essential air condition consumption , $T_{i n}$, is defined by the under limit, $T_{u n l}$, and upper limit, $T_{u p l}$, of the indoor temperature.

The HPS exploits various energy saving strategies to provide BTS energy efficiency. One of these methods is to use ECO strategy to turn radio equipment and receivers off and reduce the number of active users $N_{u}$ with the lower limit $N_{u m i n}$. There is also possibility to shifting the cooling load to a latter hour when the power consumption is low and/or there is surplus power due to generating nonrenewable sources. Cell size has strongly effect on the BTS power consumption, as the transmit power determines the range of the wireless signal. In this case, adjusting cell size can reduce or increase power consumption which is given as [8]

$$
P_{B T S}=\zeta_{1} \frac{Z}{R^{2}}+\zeta_{2} N_{u} R^{\eta}
$$

where $Z$ is an area with an homogeneous cell size, $R$ is the cell size, $\zeta_{1}$ and $\zeta_{2}$ depend on various parameters used in the model and $\eta$ is the associated path-loss exponent.

The HPS in [7] uses the nearly instantaneous balance between the total power generation $\alpha=P_{p}(t) n_{c}+P_{w}(t) n_{r}$ and the total power consumption $\beta=D(t)+A(t) / n_{i}$ as a criterion to choose different energy saving strategies at time slot $t$. Table I summarizes these strategies according to this selection criterion.

\section{B. Control and Communications System}

An intelligent control system is essential for stable and reliable operation of the BTS HPS. This system is composed of sensors, actuators, and a control unit as shown in Fig. 1. 
TABLE I

ENERGY SAVING STRATEGIES FOR THE HYBRID POWER SYSTEM ACCORDING TO BALANCE BETWEEN TOTAL POWER GENERATION $\alpha$ AND TOTAL POWER CONSUMPTION $\beta$.

\begin{tabular}{|c|c|}
\hline Case & Energy Saving Choices \\
\hline$\alpha>\beta$ & $\begin{array}{l}\text { - Charging batteries } \\
\text { - Decreasing indoor temperature } \\
\text { - Increasing the coverage area of the BTS }\end{array}$ \\
\hline$\alpha<\beta$ & $\begin{array}{l}\text { - Decharging batteries } \\
\text { - Purchasing energy from the utility } \\
\text { - Producing energy by the diesel generator } \\
\text { - Decreasing the coverage area of the BTS } \\
\text { - Shifting the cooling load to a latter hour } \\
\text { - Using ECO mode } \\
\text { - Adjusting the cell size }\end{array}$ \\
\hline$\alpha=\beta$ & - Supply locally demand and no connection to grid \\
\hline
\end{tabular}

Sensors/actuators are attached on rectifiers, converters, power generators and loads to sense and measure various parameters, such as active power and passive power for processing in control unit. The control unit performs several major functions as follows:

- Managing power generators including diesel generator, wind, solar, and battery storage to modulate their output power levels according to the power consumption of the loads;

- Determining surplus and shortage in electrical power based on the total power generation and the total loads;

- Connecting or disconnecting the hybrid system to the grid through AC switch;

- Establishing energy saving strategies defined in Table I to reduce power consumption; and

- Control and monitoring rectifiers, converters, and other sensors/actuators.

It is evident that for a reliable tight control of the system, a communications network is necessary to enable connectivity between the control unit, loads, power generators and other sensors/actuators located within BTS. Considering the application, a low-cost reliable wireless communications would be best for this case. An efficient candidate of wireless communication is wireless sensor networks based on IEEE 802.15.4 standard, using ZigBee technology. This technology is a low cost, low power that can be used for control and monitoring the HPS. Fig. 1 illustrates a ZigBee-based wireless sensor network for the HPS, where mesh topology provides high reliability communications links from power generators, rectifiers, converters, and loads to the control unit. This can be achieved through redundant paths suing mesh routers and a coordinator.

\section{The SMART Hybrid Power System}

\section{A. Real-Time Energy Management}

As previously explained, the conventional BTS HPS has the capability to connect and disconnect from the electrical grid, according to measuring shortage and surplus in electrical power. In the case of disconnection, it locally balances supply and demand, and there is no capability to sell the surplus energy to the grid. The conventional HPS also employs the power grid and local energy resources to balance supply and demand, when it is connected to the grid. In this case, there is no any intelligent connection between the utility control center and the hybrid system, that provide real-time management information such as pricing to facilitate ECS capability. As a solution to above problems, we propose the SHPS in which the BTS is equipped with an ECS unit for scheduling the power consumption. In doing so, ECS uses bidirectional communications to obtain real-time energy management information such as real-time pricing and has an ability to respond to the price in electric market.

\section{B. Real Time Pricing and Cost Optimization}

Real time pricing programs are typically an electric supply rate option offered by the utility, in which consumers pay electricity supply rates that vary by the hour or the time slot. In this case, prices are typically set over different time slots or hours in advance, and sent to the SHPS. Then, ECS can estimate the demand $\alpha$ and supply $\beta$ for a time slot and make a decision to buy or sell electricity. This can consequently facilitate the balance between supply $\alpha$ and demand $\beta$ such that the total operational cost is reduced.

Now, in order to formulate real-time cost optimization, let $g(t)$ denote the price in which the BTS buys or sells electricity to the grid at a time slot $t$. The cost of electricity provided by the diesel generator is a quadratic function of real power generation which is given by [9],

$$
d(t)=a P_{d}(t)^{2}+b P_{d}(t)+c,
$$

where $a, b, c$ are constant and defined for each time slot $t$. The amount of renewable generation of energy can be predicted for the next few minutes based on past measurements [10]. Let $\widehat{x}$ denote the expected value, denoted by $\mathbb{E}\{\cdot\}$, of $x$ for the next few minutes based on past measurements and can be defined as [11]

$$
\widehat{x}(t)=\mathbb{E}\{x(t) \mid x(t-1), x(t-2) \ldots\} .
$$

Moreover, let $\widetilde{y}$ denotes the estimated value for $y$ when $x$ takes its expected value.

At the beginning of each time slot $t$, ECS first obtain an estimate of renewable generations $\widetilde{\beta}$ using

$$
\begin{aligned}
& \widehat{P}_{w}(t)=\mathbb{E}\left\{P_{w}(t) \mid P_{w}(t-1), P_{w}(t-2) \ldots\right\}, \\
& \widehat{P}_{p}(t)=\mathbb{E}\left\{P_{p}(t) \mid P_{p}(t-1), P_{p}(t-2) \ldots\right\} .
\end{aligned}
$$

This follows by computing an estimation of demand $\widetilde{\alpha}$ through the estimation of the average values $\widetilde{A}$ and $\widetilde{D}$. Estimating the values for $\widetilde{\alpha}$ and $\widetilde{\beta}$, there are the three following cases.

1) $\widetilde{\alpha}<\widetilde{\beta}$ : By receiving real-time prices for the next $n$ time slots, the ECS attempts to schedule the energy by solving the 
following problem:

$$
\begin{array}{cl}
\min _{\widetilde{P}_{g}(t), \widetilde{P}_{d}(t)} & \sum_{t=t_{1}}^{t_{n}}\left(\widetilde{P}_{g}(t) g(t)+\widetilde{P}_{d}(t) d(t)\right) \\
\text { s.t. } & E_{m}(t) \leq 0, t=t_{1}, \ldots, t_{n} . \\
& T_{u n l}<T_{i n}(t)<T_{u p l} \\
& R(t)>R_{\text {min }} \\
& N_{u}(t)>N_{\text {umin }} . \\
& P_{b}(t) \geq 0
\end{array}
$$

In (6), $E_{m}$ can be written as

$$
\begin{aligned}
E_{m}= & \left(\widetilde{P}_{d 1}+\widehat{P}_{w}+\widetilde{P}_{g 1}\right) n_{r} \\
& +\frac{\widetilde{P}_{d 2}+\widetilde{P}_{g 2}-\widetilde{A}}{n_{i}}+\widehat{P}_{p} n_{c}-\widetilde{D},
\end{aligned}
$$

where $P_{d 1}, P_{g 1}$ represent the amount of the energy supplied by the diesel generator and the grid, respectively, for the DC part of consumption or batteries through Part 1 and $P_{d 2}, P_{g 2}$ are the energy that supply by the diesel generator and the grid, respectively, for the AC part of consumption. We also assume that $P_{b}(t) \geq 0$, which implies that there always exists a backup energy in case that there is any interruptions or shortage in power due to an power estimation error or power failure. The problem described in (6) is a convex minimization one, that can be solved using common subgradient methods that are computationally tractable.

2) $\widetilde{\alpha}>\widetilde{\beta}$ : ECS can communicate to the utility for selling electricity and/or facilitate energy saving strategies, such as charging batteries, decreasing indoor temperature, and increasing the coverage area of the BTS, as indicated in Table I.

3) $\widetilde{\alpha}=\widetilde{\beta}$ : In this case, ECS can locally balance demand and supply, without any connection to the grid for selling or buying the electricity.

The following algorithm lists the stages of execution for the real-time energy management that is performed by ECS. As

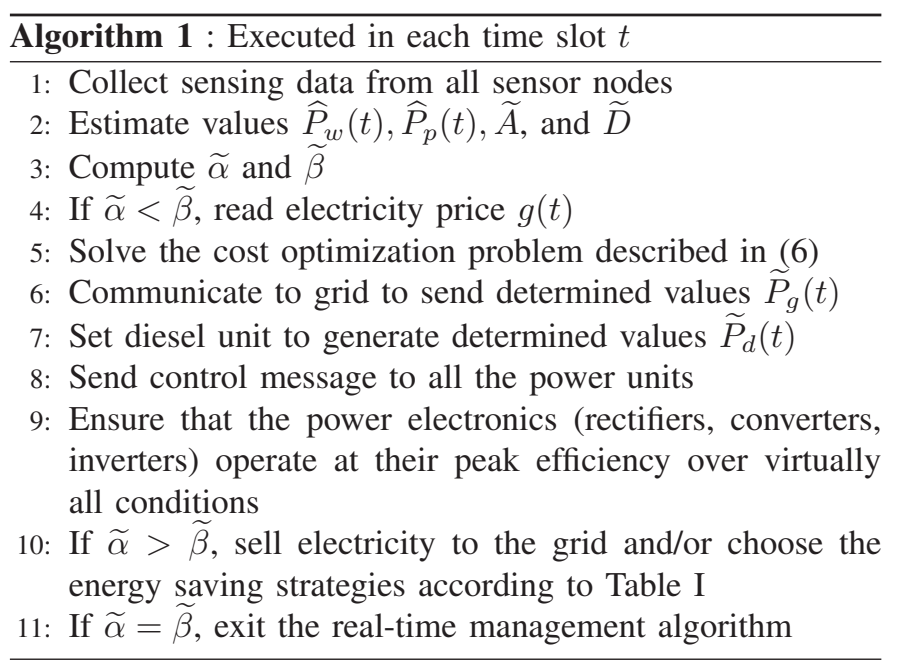

ECS determines the energy saving strategies, control message are sent to to all the power units, while it ensures that the power electronics (rectifiers, converters, inverters) operate at their peak efficiency over virtually all conditions [7].

ECS is embedded within the control unit, which requires to communicate with the utility control to obtain real-time energy management information, and also send demand estimation to the utility center. There are two possible communications with the utility center, through a smart grid communications infrastructure or a commercial network. Fig. 2 illustrates communications links between the control unit and the utility center.

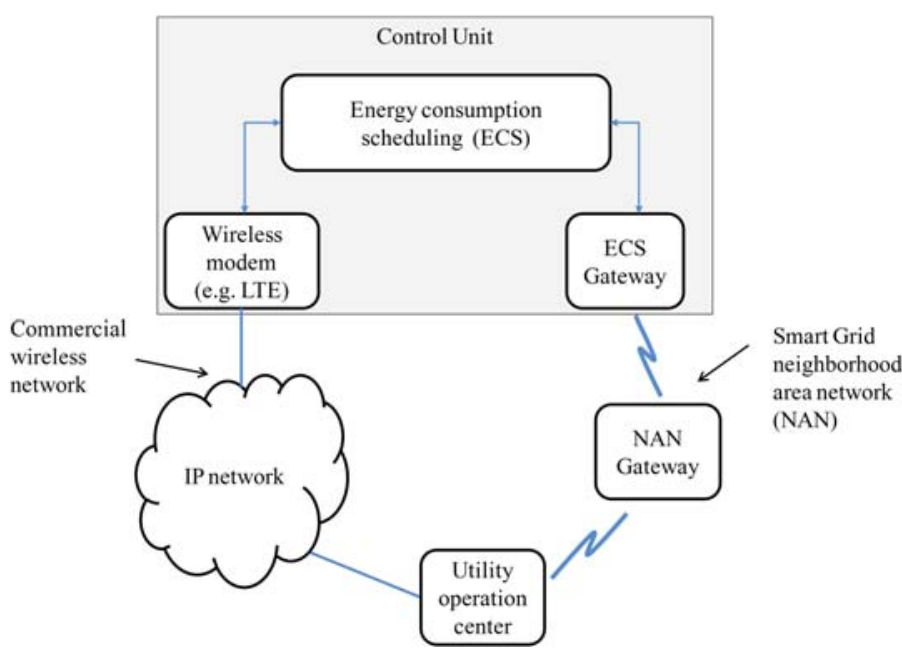

Fig. 2. The SHPS including ECS and communications links to the utility operation and control.

\section{TECHNO-ECONOMIC ANALYSIS}

In this section, we propose a sizing model for the hybrid system, based on the algorithm proposed in [7]. The cost of a hybrid system depends on the following factors: the nominal power of wind and PV power generators, the number of batteries, the life time of the hybrid system, the usage of diesel generator or grid, the replacement cost of batteries, the capital and the operational cost associated with the sensor network, and the maintenance cost of the hybrid system. From all the suitable combinations of parts of hybrid system, optimum is the one with the lower cost. The present value of the total cost can be given by

$$
V_{p}=V_{c}+V_{m}+V_{i}-V_{S}+V_{r},
$$

where $V_{c}$ is the initial capital cost of the system [7], which depends on the nominal power of wind turbines $\left(P_{w n}\right)$, the nominal power of the PV generators $\left(P_{p n}\right)$, the nominal power of diesel generator $\left(P_{d}\right)$, the number of batteries $\left(N_{b}\right)$ and their costs and the cost associated with the sensor network and the communication infrastructure. Moreover, $V_{m}$ is the present value of the total maintenance cost of the system, $V_{i}$ is the present value cost of energy which is provided from diesel generator and grid for all the years, $V_{S}$ is the present cost of the renewable energy that the BTS provides to the grid 
and $V_{r}$ is the total replacement cost for all the lifetime of the system. Loss of load probability is defined by $S$, while its upper limit is defined by $L$.

$$
\begin{array}{cl}
\min _{P_{r}, N_{p}, P_{d}} & V_{p} \\
\text { s.t. } & S \leq L \\
& C E \leq C E_{\max }
\end{array}
$$

In the previous equation $S$ can be described as

$$
S=\frac{\tau}{T},
$$

where $T$ is the number of hours in this study with weather data input and

$$
\begin{array}{r}
\tau=\sum_{t=0}^{T} \mathscr{D}\left[\left(P_{d 1}+P_{w}+P_{g 1}\right) n_{r}+P_{p} n_{c}\right. \\
\left.+\frac{P_{d 2}+P_{g 2}-A}{n_{i}}-D<0\right] .
\end{array}
$$

In (10), $\mathscr{D}[q]$ denotes the time duration where the $q$ event is true. Moreover, $C E$ is the carbon emissions, while $C E_{\max }$ is its upper limit.

Table II summarizes a techno-economic analysis with various configurations for the SPHS, using the real time prices and the hourly weather data used in [7]. We consider the lifetime of the system equal to 25 years, while we set $L=0$.

TABLE II

A TECHNO-ECONOMIC ANALYSIS (IN DOLLARS) WITH VARIOUS CONFIGURATIONS FOR THE SMART POWER HYBRID SYSTEM.

\begin{tabular}{|l|l|l|l|l|l|l|l|}
\hline$P_{p n}$ & $P_{w n}$ & $V_{c}$ & $V_{m}$ & $V_{i}$ & $V_{s}$ & $V_{r}$ & $V_{p}$ \\
\hline 1 & 1 & 5000 & 200 & 1341 & 943 & 500 & 6098 \\
\hline 1 & 2 & 7000 & 200 & 884 & 6254 & 500 & 2330 \\
\hline 2 & 1 & 6000 & 200 & 1031 & 3939 & 500 & 3792 \\
\hline 2 & 2 & 8000 & 200 & 646 & 9279 & 500 & 67 \\
\hline
\end{tabular}

Moreover, we found that by increasing the nominal power of the renewable generators, we could further reduce $V_{p}$ and even make a profit.

\section{NumericAl RESUlTS AND DisCUSSION}

In this section, numerical and simulation results are presented to demonstrate the performance of the proposed smart hybrid method. More specifically, the operational cost and the demand and supply estimation are evaluated along with the optimized power consumption achieved. For the purpose of this study, we use the actual hourly real time prices adopted by the Illinois power company [12], as shown in Fig. 3. Without loss of generality, we assume a diesel generator with constant parameters $a=0.002, b=0.005, c=0.015$ and rectifiers and converters with parameters $n_{i}=n_{r}=n_{c}=1$. We also assume that the SHPS sells energy to the grid at the same price as it costs for buying electricity from the grid. The hourly prices of electricity are updated every one hour for the time horizon of two hours, while the SHPS executes Algorithm 1 every one hour. Further, it is assumed that half of the cooling

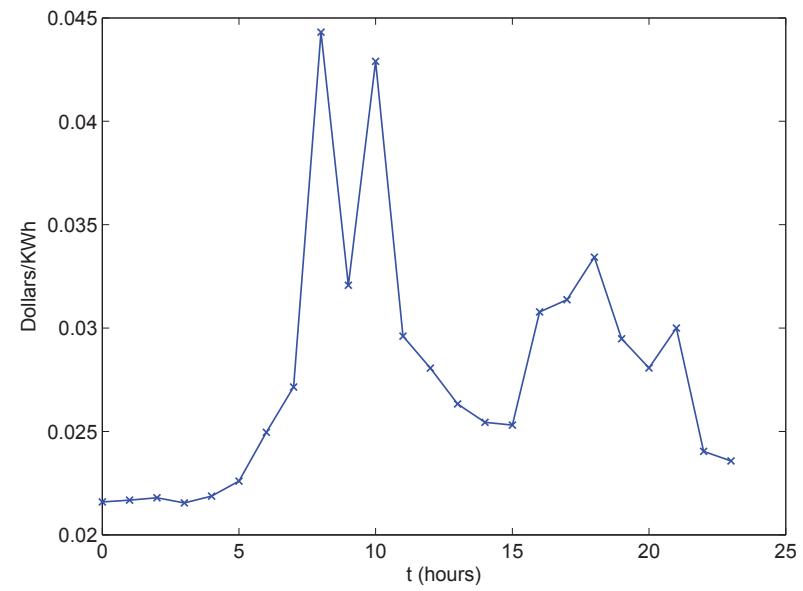

Fig. 3. The actual hourly real time prices for 24 hours daily measured in $2 / 1 / 2013$ from the Illinois power company [12].

load from the present hour can be shifted to the next hour, if the electricity price, $g$, is lower.

Fig. 4 shows the actual average of renewable power generations $(\alpha)$ and BTS power consumption $(\beta)$ in comparison with the estimated value of renewable power generations and BTS power consumption. Both estimated and actual values for power consumption are almost the same, while these amounts are slightly different for renewable power generations. This was expected due to the variable nature of renewable energy sources. As a result, the estimation error can have very small impact on the performance of the proposed algorithm in terms of the cost of operations.

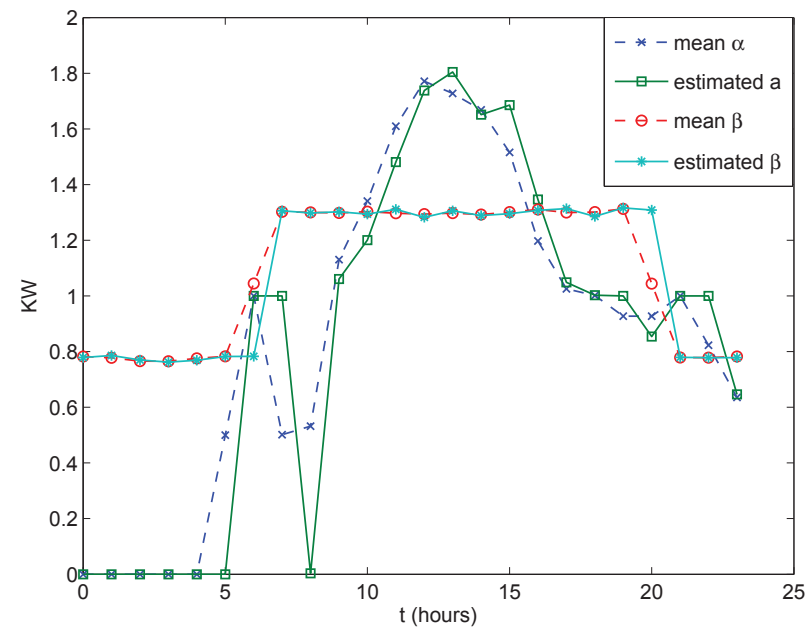

Fig. 4. The actual average of renewable power generations $(\alpha)$ and power consumption $(\beta)$ in comparison with the estimated value of renewable power generations and power consumption.

Fig. 5 presents the estimated value of renewable power generations and the optimized values of BTS power consumption using the SHPS. This also includes the estimated value of diesel generation and the amount of electricity supplied by the grid. The results show that the proposed method perfectly 
balance estimated demand $(\beta)$ and supply $(\alpha)$, whenever there is a shortage through using diesel generators or the power grid. When there is a surplus energy, e.g. between $t=10$ and $t=16$, the SHPS sells electricity to the grid, representing with negative value of energy shown in Fig. 5. The results also show that the smart hybrid system rely on diesel generator for a short periodic of time $(t=8)$, in which $g(8)>d(8)$, to compensate a shortage in energy which can have very low $\mathrm{CO} 2$ emissions, while by efficiently using the renewable energygiving the surplus to the grid-the total $\mathrm{CO} 2$ emissions are minor.

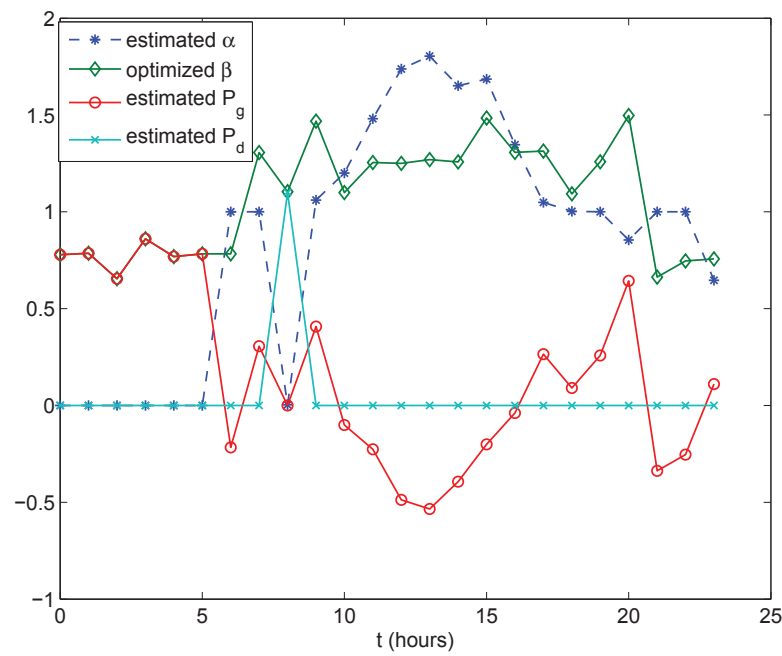

Fig. 5. The estimated renewable power generations and the optimized BTS power consumption, the estimated diesel power generations and energy required from the grid with using the SHPS.

Fig. 6 shows the hourly operational cost of the BTS system for three difference cases: 1) the HPS only use diesel power generator for supplying shortage in power, 2) the HPS only uses the grid for supplying shortage in power, and 3) the SHPS balance demand and supply using real-time energy management, while it uses both the grid and the diesel generator.

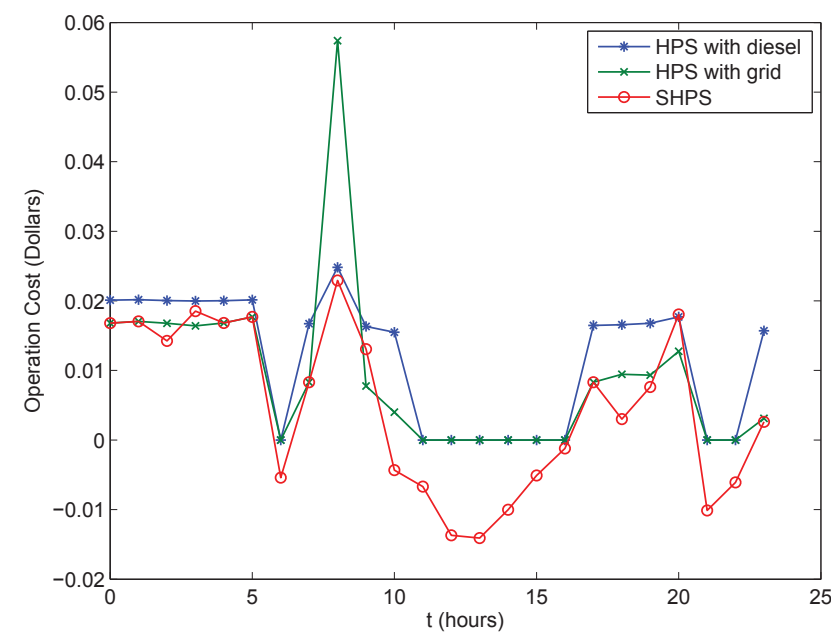

Fig. 6. The hourly operational cost for HPS uses only diesel power generator and HPS uses only the grid, and for the SHPS with real-time energy management.
As we can see in Table III which summarizes the daily cost of the three systems, the daily operational cost of the SHPS is about $61 \%$ and $51 \%$ significantly reduced, comparing with the daily operational cost of both cases for the HPS.

TABLE III

A COMPARISON OF DAILY OPERATIONAL COST FOR THE HPS ONLY USE DIESEL POWER GENERATOR, THE HPS ONLY USES THE GRID AND THE SHPS.

\begin{tabular}{|l|l|}
\hline System Configuration & Daily Cost in Dollars \\
\hline HPS with diesel & 0.2770 \\
\hline HPS with grid & 0.2219 \\
\hline SHPS & 0.1082 \\
\hline
\end{tabular}

\section{CONCLUSiOnS}

In this paper, we proposed a smart hybrid power system (SHPS) for a BTS taking real-time energy management into account. In this regard, we developed a sensor control system and a wireless sensor network for the hybrid power system (HPS). A real-time energy management algorithm was proposed to reduce the cost of BTS operation according to realtime pricing, demand and supply estimation. This can provide the HPS with the ability to selling and buying electricity from the power grid. Simulation results verified that the proposed smart hybrid method can remarkably reduce the operational cost of the BTSs. A techno-economic analysis was performed to obtain the total cost of the hybrid power system according to the cost associated with the sensor network and communications infrastructure, and real-time energy pricing.

\section{REFERENCES}

[1] W. Vereecken, W. Van Heddeghem, M. Deruyck, B. Puype, B. Lannoo, W. Joseph, D. Colle, L. Martens, and P. Demeester, "Power consumption in telecommunication networks: overview and reduction strategies," IEEE Commun. Mag., vol. 49, no. 6, pp.62-69, June 2011.

[2] S. N. Roy, "Energy logic: a road map to reducing energy consumption in telecommunications networks," in Proc. IEEE International Telecommunications Energy Conference (INTELEC), Sept. 2008.

[3] Ericsson, "Sustainable energy use in mobile communications," White Paper, Aug. 2007.

[4] R. Tu, X. H. Liu, Z. Li, and Y. Jiang, "Energy performance analysis on telecommunication base station," Elsevier, Energy and Buildings, vol. 43, no. 2-3, pp.315-325, Feb.-Mar. 2011.

[5] S. Hashimoto, T. Yachi, T. Tani, "A new stand-alone hybrid power system with wind generator and photovoltaic modules for a radio base station," in Proc. IEEE International Telecommunications Energy Conference (INTELEC), pp. 254-259, Sept. 2004.

[6] P. Nema, R.K. Nema, S. Rangnekar, "Minimization of green house gases emission by using hybrid energy system for telephony base station site application," Elsevier, Renewable and Sustainable Energy Reviews, vol. 14, no. 6, pp. 1635-1639, Aug. 2010.

[7] P. D. Diamantoulakis and G. K. Karagiannidis, "On the design of an optimal hybrid energy system for base transceiver stations," Journal of Green Eng., vol. 3, no. 2, pp. 127-146, 2013.

[8] S. Bhaumik, G. Narlikar and S. Chattopadhyay, "Breathe to stay cool: adjusting cell sizes to reduce energy consumption," in Proc. ACM SIGCOMM Workshop on Green Networking, Aug-Sep. 2010.

[9] Y. Hong, R. Lian, "Optimal sizing of hybrid wind/PV/diesel generation in a stand-alone power system using Markov-based genetic algorithm,' IEEE Trans. on Power Delivery, vol. 27, p.640-647, 2012.

[10] M. Ghamkhari and H. Mohsenian-Rad, "Optimal integration of renewable energy resources in data centers with behind-the-meter renewable generator," in Proc. IEEE International Conference on Communications (IEEE ICC), June 2012.

[11] M. Lange and U. Focken, Physical approach to short-term wind power prediction, Berlin, Germany: Springer-Verlag, 2005.

[12] https://www2.ameren.com/RetailEnergy/realtimeprices.aspx, Jan. 2013. 